\title{
Calculation and Experimental Study of Heat Exchange in a System of Plane-Parallel Channels with Surface Intensifiers
}

\author{
S. I. Kaskov
}

This paper presents the results of numerical investigation, calculation analysis and experimental study of heat exchange in a system of plane-parallel channels formed by rectangular fins, which are applied in a heat removal device using heat tubes for power semiconductor energy converters. Passive cooling (heat removal by radiation and natural convection) and active cooling (heat removal by radiation and forced convection) are investigated for various velocities of air cooling of fins by spherical vortex generators applied to its surface. A comparative analysis of the results is carried out for the average effective heat removal resistance and for the average temperature at the ends of the fins. The application of numerical modeling to solve such problems confirms the effectiveness of computational technologies. The difference between the results of the study ranges from 10 to $16 \%$ depending on the airflow rate.

Keywords: vortex enhancement, heat transfer, cooling of power electronics

\section{Introduction}

Powerful semiconductor electric energy converters remain an important component of systems for transformation and conversion of flows of energy $[1,2]$ used in nuclear power plants, airdromes, railroad transport, subway traction substations, marine vessels, in ground tests of equipment for space exploration and remain an indispensable element in designing the flowsheets of promising thermonuclear facilities.

The vector of miniaturization of electronic systems slows down, but their capacity grows appreciably, which leads to an increase in heat release and operating temperatures in them. This can be seen from the dynamics of the values of specific heat amount generated per unit volume or per surface area of the electronic device. The current trend of the development of electronics is to increase specific heat flows in cooling systems, which will be comparable in magnitude

Received March 05, 2021

Accepted June 02, 2021

Sergey I. Kaskov

kaskovsi@bmstu.ru

Bauman Moscow State Technical University

ul. 2-ya Baumanskaya 5, 1, Moscow, 105005 Russia

RUSSIAN JOURNAL OF NONLINEAR DYNAMICS, 2021, 17(2), 211-220 
with individual specific heat flows of industrial nuclear reactors and experimental facilities for investigation of thermonuclear processes and for modeling of plasma processes [3-7].

Heat release in the electronic component is defined as the product of voltage and current strength, which are fed to the device at the ultimate load, with allowance for the safety factor in developing or updating the electronic basis and in meeting the requirements concerning dimensions and weights. The cooling system must fit in the outer dimensions of electronic equipment and must not lead to a considerable increase in weight. One of the rational solutions is to use natural convective cooling systems and, as an immediate alternative, devices with forced coolant flow $[8-10]$.

\section{Review}

Structured surface elements - vortex generators of different forms which are applied on heat-exchange surfaces - are promising means for heat transfer intensification in cooling systems of radioelectronic and power equipment and in channels of various power-generating equipment and transportation facilities [11-13]. This type of heat transfer intensifiers destroys the laminar boundary layer or the viscous sublayer of the turbulent boundary layer without affecting the main flow and ensures a high thermal and thermohydraulic efficiency [14, 15]. The flow past dimples of variable depth under different flow conditions has special features due to the fact that tornado-like vortex structures have two orientations of their axes at the exit from the surface dimple: to the left or to the right, or they are in the stage of change of orientation. Each such state near one dimple of the heat-exchange surface has an effect on the state of the vortex structure of the downstream dimple. The interacting tornado-like elements of the flow are selforganizing structures [16-18]. The main feature of self-organization is the formation of complex structures from simple ones in the absence of specific influences determining this formation. A complex structure in this case is a group of tornado-like structures whose states are matched to each other as a result of the interaction. The three-dimensional tornado-like structures generated by the dimples change their states in a coordinated fashion. The parameters of the surface relief do not influence this specifically or immediately since they do not prescribe the vortex structures to deviate in a coordinated fashion in one or the other direction. The matching of states is due to nonlinearity of the equations of motion of the continuous medium, which also manifests itself when large-scale vortex structures [19, 20] arise.

Systematic research carried out over many years into the intensification of heat exchange on structured surfaces with ordered vortex generators has led to breakthrough solutions of the thermophysical problem, which combine a high thermal efficiency and a moderate growth of hydraulic losses, and to the creation of heat-exchange devices for industrial purposes [21-24]. Heatexchange surfaces with annular protuberances and cylindrical grooves, oval trench-like dimples, hemispherical protuberances and depressions [25-28], spiral and pin fins [29] have been investigated.

The variety of outward shapes, transverse and longitudinal sections of vortex generators stems from the particularities inherent in the manufacture of such structures with the necessity of their optimization to ensure specific operational characteristics. The shape of the cross-section plays a special role in letting the fluid or gas flow pass through the surface structure in cooling systems with different types of heat carriers. This explains the large diversity of shapes of vortex generators used in single-phase cooling systems, as opposed to structures for cooling with phase transition of the heat carrier. The shapes, sections and the regularity of the structure of such structured elements affect both the hydraulic losses (when the flow passes through the 
structure) and the temperature of the element to be cooled. This often makes it impossible to formulate universal recommendations, and shows that the ratios of the cumulative quality indices of structures of different shapes vary depending on the parameters of the flow of the heat carrier.

\section{Object of study}

The object of study is the cooling system of an electronic device which is depicted in Fig. 1a. The main element of the cooling system is an electric heater which imitates the operation of the electronic device (thyristor) and is an industrially manufactured AC heater based on spiral wire. The power of the heater is $720 \mathrm{~W}$. The limit voltage supplied to the heater is $85 \mathrm{~V}$. The base of the cooling system with two heat tubes of the same size is screwed up to the heater by means of bolted connections. The heat tubes have a porous wick, and water serves as working substance.

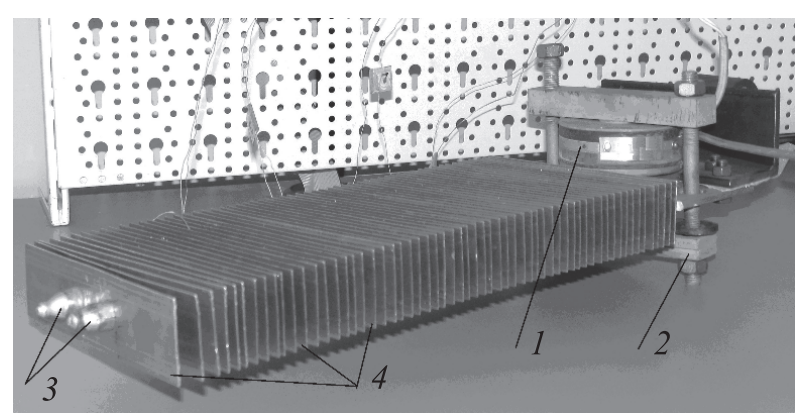

(a) 1 - electric heater (thyristor simulation); 2 - bolted connection; 3 - heat tube; 4 smooth surface fins

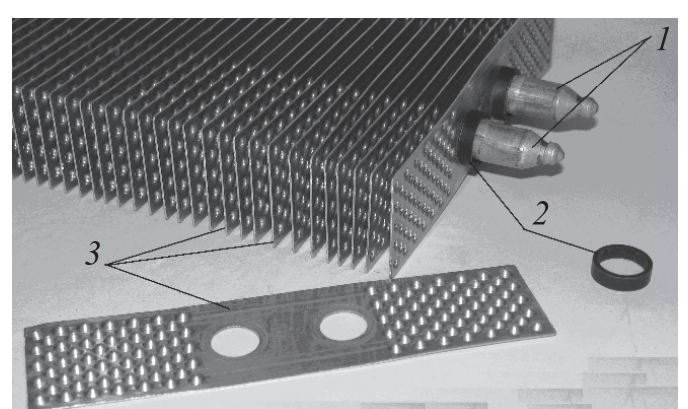

(b) 1 - heat tube; 2 - spacer washer; 3 - fins with surface dimples/protuberances

Fig. 1. Cooling system of an electronic device: (a) with smooth plat fins; (b) with spherical dimples/protuberances on flat fins.

The cooling system is a device based on heat tubes, with their ends pressed into a solid base (Fig. 1a), on which a cooled electric heater is installed by means of a special clamping device. Smooth aluminum fins and fins with hemispherical dimples/protuberances are pressed onto the heat tubes. The fins are cooled with air flow (Fig. 1b). The packing density of the hemispherical elements is 25, 50 and $75 \%$ (Fig. 3). The cooling system ensures removal of heat power of 200 to $400 \mathrm{~W}$ and has the following overall dimensions (width/height/length): 170/42/380 mm. The heat tubes are $14 \mathrm{~mm}$ in diameter and $500 \mathrm{~mm}$ in length. The dimensions of the fins (width/height/thickness) are $170 / 42 / 1 \mathrm{~mm}$. The number of fins is 60 ; the fin spacing is $6 \mathrm{~mm}$ (Fig. 2). The temperature of the heater was maintained at 52 to $60^{\circ} \mathrm{C}$.

\section{Mathematical model}

The solution is based on a mathematical model consisting of the continuity equation (4.1), the system of Reynolds equations (4.2), the state equation (4.3), the equation of energy for the heat carrier (4.4), and the equation of thermal conductivity for a rigid body, which close this system of boundary conditions and equations describing the turbulence model. The following assumptions are made: the problem is stationary; air is the working substance assumed to be Newtonian, viscous and incompressible medium; the thermophysical properties of the flow and the rigid body are taken to be constant. 


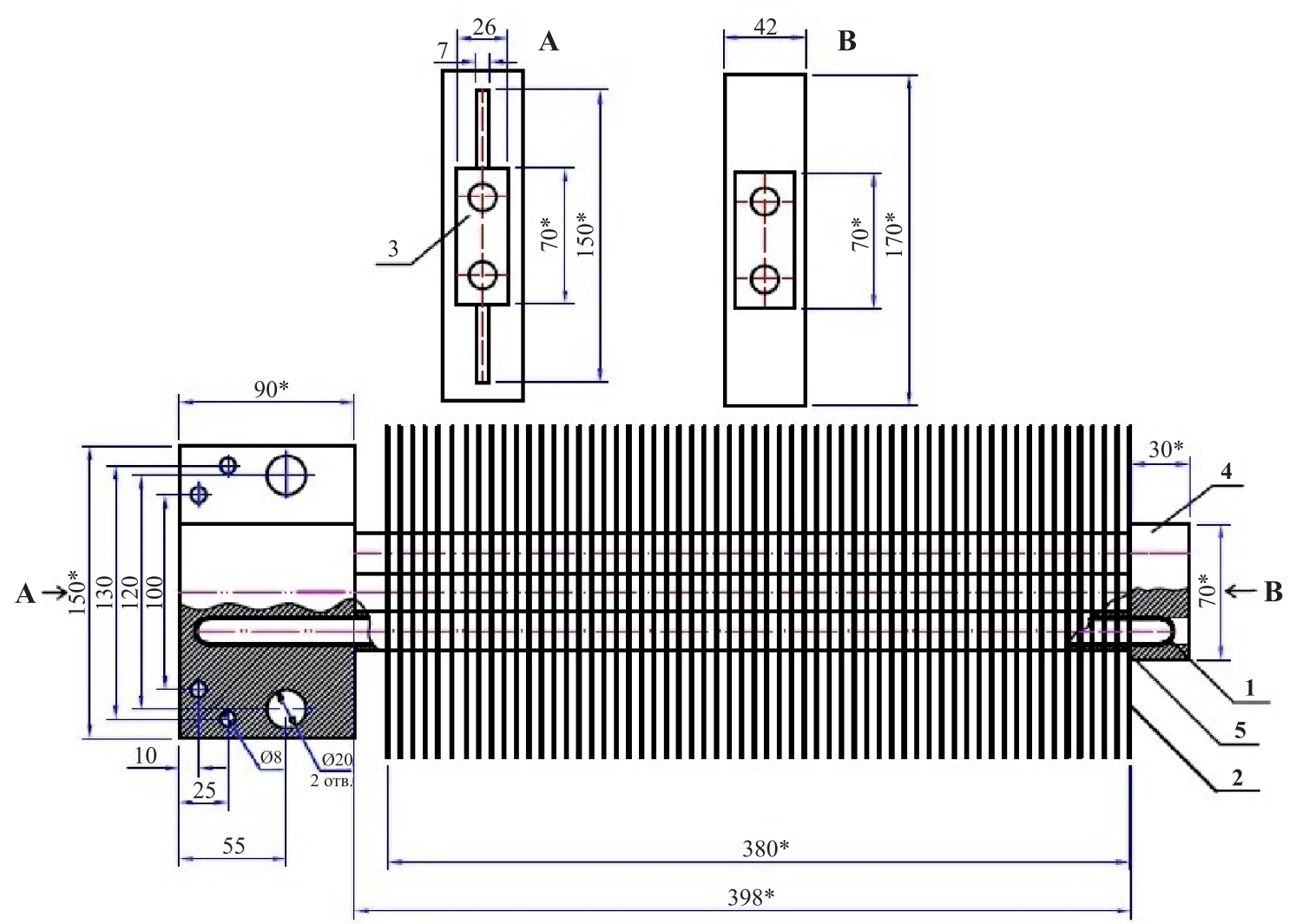

Fig. 2. Scheme of the cooling system based on finned heat tubes: 1 - heat tube; 2 - fins; 3 - base; 4 - flange; 5 - spacer washer

The system of equations takes the following forms:

the continuity equation:

$$
\frac{\partial \rho_{f} u_{j}}{\partial x_{j}}=0
$$

the equation of motion:

$$
\frac{\partial}{\partial x_{j}}\left(\rho_{f} u_{j} u_{i}-\tau_{i j}\right)=-\frac{\partial p}{\partial x_{j}}
$$

where

$$
\tau_{i j}=\left(\mu+\mu_{t}\right)\left(\frac{\partial u_{i}}{\partial x_{j}}+\frac{\partial u_{j}}{\partial x_{i}}\right)-\frac{2}{3}\left(\mu+\mu_{t}\right) \frac{\partial u_{k}}{\partial x_{k}} \delta_{i j}-\frac{2}{3} \rho k \delta_{i j} ;
$$

the equation of energy for air:

$$
\frac{\partial}{\partial x_{j}}\left(\rho_{f} h_{f} u_{i}-\left(\lambda_{f} \frac{\partial T_{f}}{\partial x_{j}}+\frac{\mu_{t}}{\sigma_{t}} \frac{\partial h_{f}}{\partial x_{j}}\right)\right)=u_{j} \frac{\partial p}{\partial x_{j}}+\tau_{i j} \frac{\partial u_{i}}{\partial x_{j}} ;
$$

the equation of thermal conductivity for the fin:

$$
\frac{\partial}{\partial x_{j}}\left(\lambda_{C} \frac{\partial T_{C}}{\partial x_{j}}\right)=0
$$




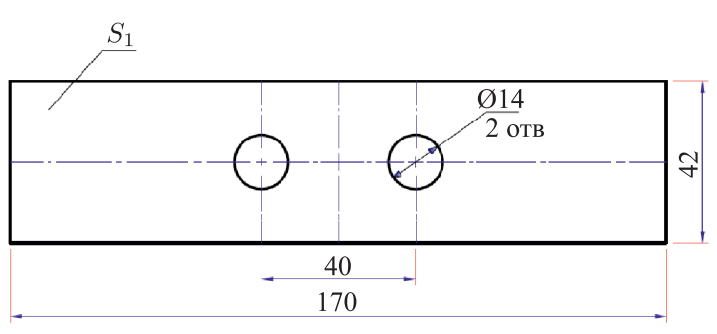

(a)

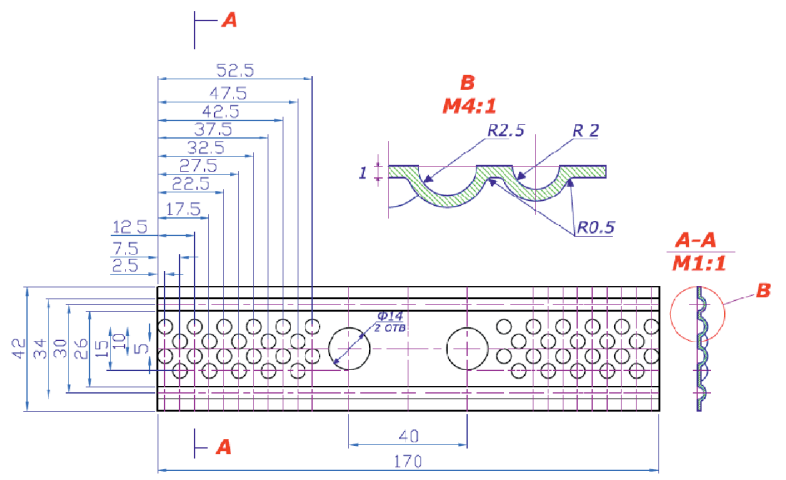

(c)

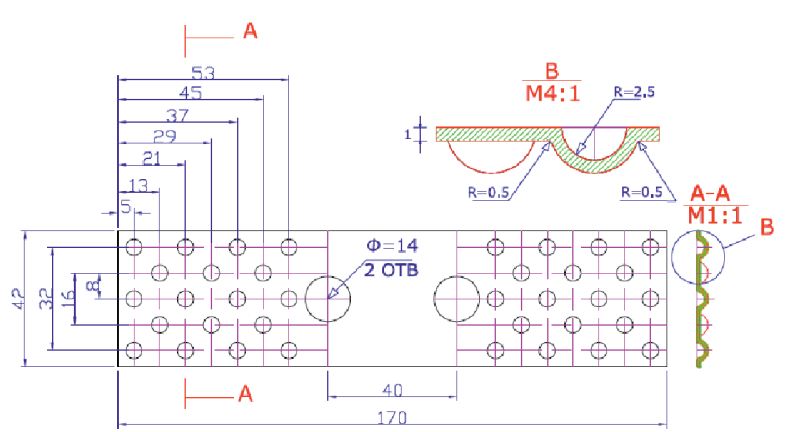

(b)

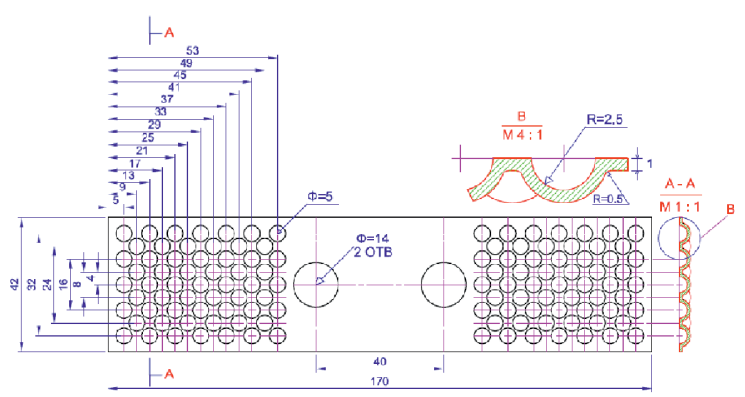

(d)

Fig. 3. Scheme of the fins of the cooling system: a) smooth flat fins; flat fins with spherical dimples/protuberances and a packing density of b) $25 \%$ c) $50 \%$ d) $75 \%$

For modeling of the processes of turbulent transfer, the two-parameter $k$ - $\omega$ low-Reynolds model has been chosen. The use of this model has been validated by the investigations and calculations presented in $[30,31]$. The equations describing the turbulent transfer (with the assumptions taken into account) are:

- the equation for the kinetic energy of turbulence:

$$
\frac{\partial}{\partial x_{j}}\left[\rho u_{j} k-\left(\mu+\frac{\mu_{t}}{\sigma_{k}^{\omega}}\right) \frac{\partial k}{\partial x_{j}}\right]=\mu_{t} P-\rho \beta^{*} k \omega ;
$$

- the equation for the velocity of dissipation:

$$
\frac{\partial}{\partial x_{j}}\left[\rho u_{j} \omega-\left(\mu+\frac{\mu_{t}}{\sigma_{\omega}^{\omega}}\right) \frac{\partial \omega}{\partial x_{j}}\right]=\alpha \frac{\omega}{k} \mu_{t} P-\rho \beta \omega^{2}+\rho S_{\omega},
$$

where $\alpha=0.52, \beta_{0}=0.072, \beta_{0}^{*}=0.09, \sigma_{k}^{\omega}=0.5, \sigma_{\omega}^{\omega}=0.5$ are empirical constants; $\beta=\beta_{0} f_{\beta}, \beta^{*}=\beta_{0}^{*} f_{\beta}^{*}, f_{\beta}=\frac{1+70 \chi_{\omega}}{1+80 \chi_{\omega}}, \chi_{\omega}=\left|\frac{\Omega_{i j} \Omega_{i k} S_{k i}}{\left(2 \beta_{0}^{*} \omega\right)^{3}}\right|, \Omega_{i j}=\frac{\partial u_{i}}{\partial x_{j}}-\frac{\partial u_{j}}{\partial x_{i}}, f_{\beta}^{*}=1, \chi_{k} \leqslant 0 ;$ $f_{\beta}^{*}=\frac{1+680 \chi_{k}^{2}}{1+400 \chi_{k}^{2}}, \chi_{k}>0, \chi_{k}=\frac{1}{\omega^{3}} \frac{\partial k}{\partial x_{j}} \frac{\partial \omega}{\partial x_{j}}, S_{\omega}=0$. The turbulent viscosity is defined by the relation $\mu_{t}=\rho \frac{k}{\omega}$. 
For numerical modeling of the plane-parallel channels, based on fins with surface intensifiers, and for development of the solid three-dimensional design model of the element of the cooling system, the Unigraphics SolidWorks 2013 3D CAD software was used. The SIMPLE procedure was used to solve the Reynolds equations. The ICEM CFD software was used to create a grid. Test calculations of network convergence were performed, which determined the choice of the number of nodes and elements. Numerical modeling of the flow and heat exchange for the design model was performed in the ANSYS - Fluid Flow CFX version 14.5. The type of the network chosen was Hexahedra mesh. The network contained 558584 nodes and 501176 elements. The design model with the network and the boundary conditions is shown in Fig. 4. The boundary conditions were given at the entrance, exit and on the side walls of the control volume. By design, all undefined boundaries were rigid, impermeable walls. The entry and exit boundary conditions were open and symmetric, respectively. The dimensions were: $\min x, \max x$ : $-0.0866093 \mathrm{~m}$, $0.0833907 \mathrm{~m} ; \min y, \max y:-0.0266057 \mathrm{~m}, 0.0573943 \mathrm{~m}$; $\min z, \max z: 0.0083 \mathrm{~m}, 0.0251 \mathrm{~m}$; the ratio of the maximum length was 16.4817 . The volume was $30.000236119 \mathrm{~m}^{3}$.
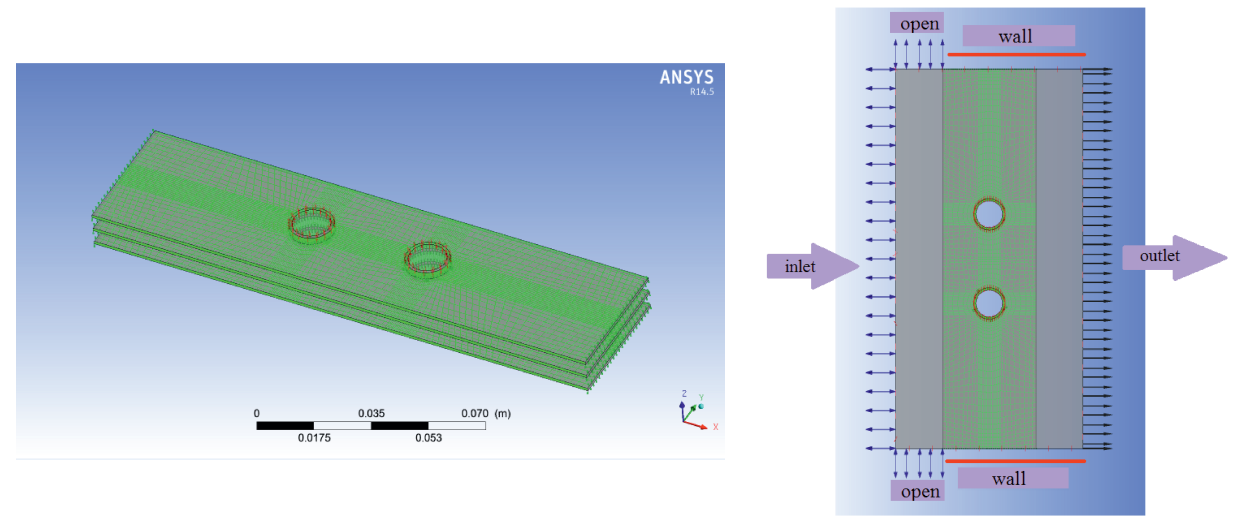

Fig. 4. Design model with network.

Physical domain of the model. A stationary problem is considered, the model includes six domains: domain - AIR. The following characteristics are given: the type of fluid — air, which is an ideal gas; the air pressure at the entrance is atmospheric (barometric, $100000 \mathrm{~Pa}$ ); the model of heat transfer is the density of heat flow from the tubes; the turbulence model is a low-Reynolds model. Domain - FINS. The following characteristics are given: domain - rigid, impermeable, without motion; material - aluminum; domain — TUBES. The following characteristics are given: domain - rigid, impermeable, without motion; material - copper. The interface of the domain is a domain of interaction of air and tubes. The interface of the domain is a domain of interaction of air and fins. The interface of the domain is a domain of interaction of the fins and the tubes.

Physical boundary conditions of the model. This model has three boundary conditions: tube_source_1 and boundary conditions, tube_source_2 and the density of heat flow from the heat tubes to the fins $q=7479.08 \mathrm{~W} / \mathrm{m}^{2}$, which is constant for all regimes. Entry cross-section: (Boundary - OPENING).

The air temperature at the entrance into the model $T_{B} .5$ temperature values were considered: $T_{B 1}=17.8^{\circ} \mathrm{C}, T_{B 2}=17.9^{\circ} \mathrm{C}, T_{B 3}=16.4^{\circ} \mathrm{C}, T_{B 4}=17.6^{\circ} \mathrm{C}, T_{B 5}=17.3^{\circ} \mathrm{C}$. The temperature values were taken from the experimental conditions for subsequent comparison of experimental and numerical data. Exit cross-section: (Boundary-OUTLET). Air velocity at the exit from the model $w .5$ values of air velocity were considered: $w_{1}=0.348 \mathrm{~m} / \mathrm{s}, w_{2}=0.683 \mathrm{~m} / \mathrm{s}$, 
$w_{3}=0.946 \mathrm{~m} / \mathrm{s}, w_{4}=1.217 \mathrm{~m} / \mathrm{s}, w_{5}=1.487 \mathrm{~m} / \mathrm{s}$. The power of the heat flow withdrawn from the simulator of the semiconductor converter was set to be constant for all regimes and took the values $Q=250 \mathrm{~W}$.

\section{Results}

When the numerical modeling was performed, the distributions of the temperatures of fins and air and the velocities of air flow and pressures were obtained for each operation mode of the cooling system. Figure 5 presents the results of numerical modeling for the first mode of operation.

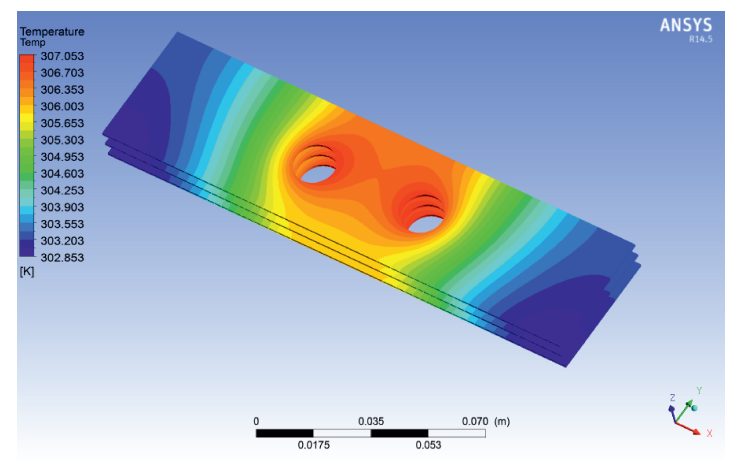

(a)

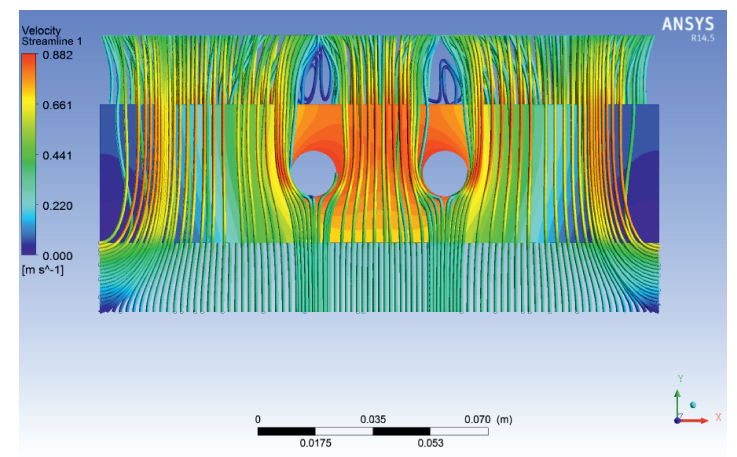

(c)

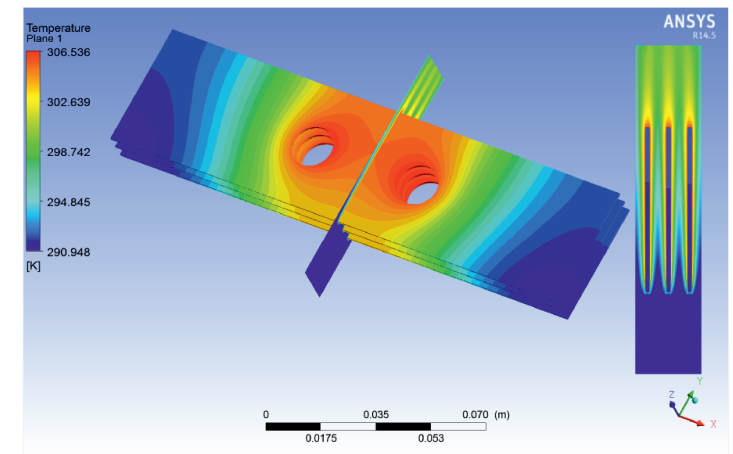

(b)

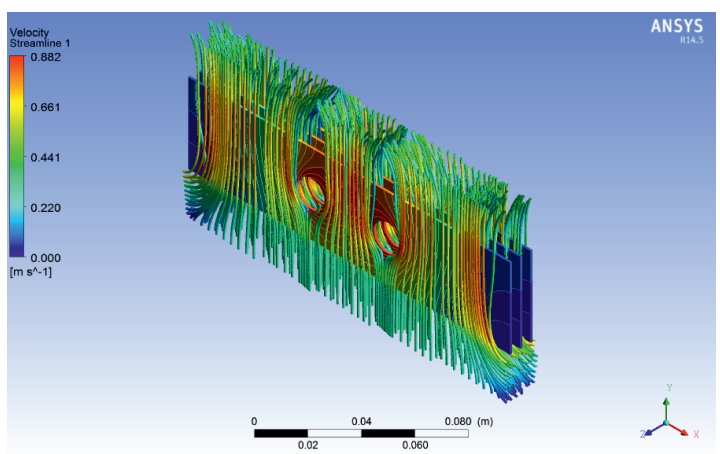

(d)

Fig. 5. Results of numerical modeling a) distribution of the temperature of fins; b) distribution of the temperature of fins and air; c) distribution of the flow velocity; d) streamlines in the interfin space.

\section{Conclusions}

The comparative analysis of the previously obtained experimental and calculated results $[32,33]$ with numerical studies was carried out for the average temperature at the ends of the fins. The analysis was performed for 5 regimes of flow past the finned heat tubes, which differed in the air flow velocities and were characterized by the same values of heat flow. The results of experimental and numerical investigations and calculation studies for the average temperature at the ends of the fins are presented in Fig. 6a, and the comparative analysis of the results of experimental, numerical and calculation studies was carried out also for the average 


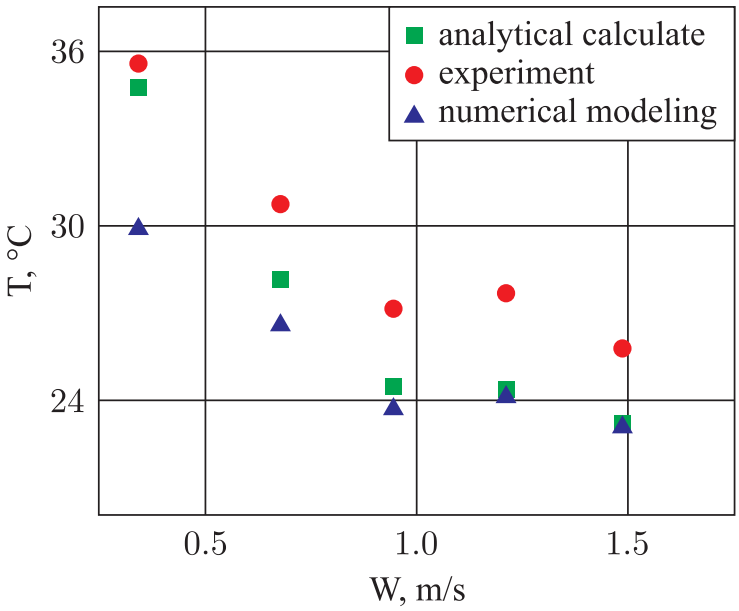

(a)

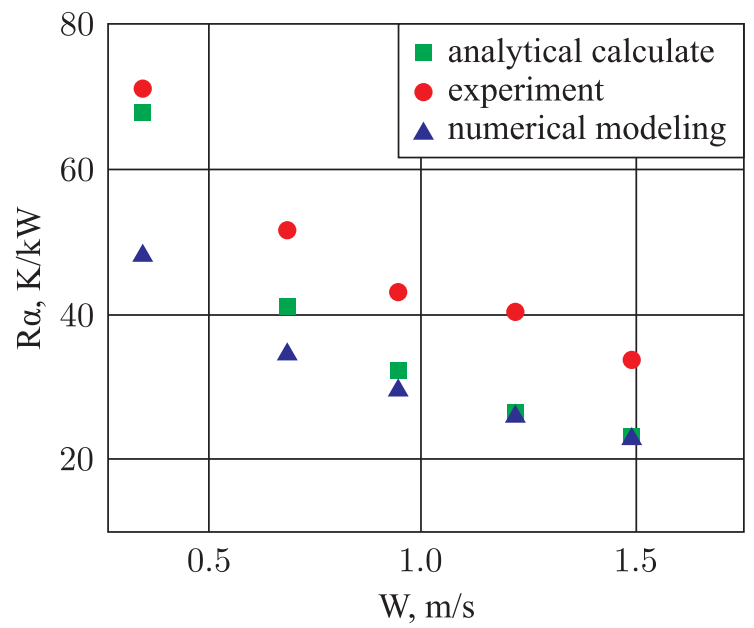

(b)

Fig. 6. Comparative analysis of the results of experimental, numerical and calculation studies a) for the average temperature at the ends of the fins b) for the average "effective" heat removal resistance.

"effective" heat removal resistance $R_{\alpha}$ (the ratio between the average temperature at the ends of the fins $T_{r}$ and the temperature of the incident air flow $T_{w}$ to the heat flow). The results of experimental investigations, numerical modeling and analytical estimation of calculation studies concerning the average temperature at the ends of the fins are presented in Fig. $6 \mathrm{~b}$ for the most effective packing density of the surface intensifiers: $50 \%$.

The data analysis has revealed that the deviations of the calculated, experimental and numerical results concerning the average temperature at the ends of the fins range from 10 to $16 \%$. At small flow rates the calculated values agree well with the experimental results, and at flow rates of order $1.5 \mathrm{~m} / \mathrm{s}$ the results of the numerical investigation and those of the calculation study are almost the same.

The data analysis has shown that the deviations of calculated, experimental and numerical results concerning the average "effective" heat removal resistance range from 31 to $36 \%$. At small flow rates, the calculated values agree well with the experimental results, and at flow rates of order $1.5 \mathrm{~m} / \mathrm{s}$ the results of the numerical investigation and those of the calculation study are almost the same.

The discrepancy between the experimental data is mainly due to the level of the velocities considered. The velocities from 0.348 to $1.5 \mathrm{~m} / \mathrm{s}$ are close to transient flows. In the calculation studies, dependences for the laminar flow were used. Therefore, at low velocities the experimental and calculated values coincide. In calculations for increasing velocities (transient flow regimes) it would be necessary to pass to dependences for the transient regime. This would increase the coefficients of heat transfer and reduce the level of discrepancy between the calculated and experimental data.

The difference between the numerical and experimental data is mainly due to possible differences between the conditions of numerical and experimental investigations: in the numerical investigations, a small (1/20 part) element (domain) of the cooling system was considered and no account was taken of a possible irregularity of air flow to the surface of the fins in the entire cooling system, irregularity of the temperatures of the base along the length of the heat tubes and fins and other conditions that were not taken into account in the model. 


\section{References}

[1] Tretiyakov, G.A., Dilevskaya, E. V., and Bryanzev, A. V., Thermal Calculation of Powerful AirCooled Converters, Moscow: Energoatomizdat, 1986 (Russian).

[2] Dilevskaya, E. V., Kaskov, S. I., and Leontyev, A. I., Investigation of Thermal Conditions of Powerful Solid-State Power Transformers, Vestn. Mezhdunarodn. Akad. Kholoda, 2008, no.4(29), pp. 34-38 (Russian).

[3] Mitrofanova, O. V. and Ivlev, O.A., The Effect of Flow Swirling on the Safety and Reliability of Nuclear Power Installations of New Generation, in Proc. of the 6st Internat. Conf. "Heat and Mass Transfer and Hydrodynamics in Swirling Flows" (Novosibirsk, 2017), p. 23.

[4] Dedov, A. V., Challenges for the Heat Transfer Enhancement in Thermonuclear Sources of Neutrons and Fusion Reactors, in Proc. of the 6st Internat. Conf. "Heat and Mass Transfer and Hydrodynamics in Swirling Flows" (Novosibirsk, 2017), p. 15.

[5] Kuzenov, V.V., Ryzhkov, S.V., and Starostin, A. V., Development of a Mathematical Model and the Numerical Solution Method in a Combined Impact Scheme for MIF Target, Russian J. Nonlinear Dyn., 2020, vol. 16, no. 2, pp. 325-341.

[6] Ryzhkov, S. V. and Kuzenov, V. V., New Realization Method for Calculating Convective Heat Transfer near the Hypersonic Aircraft Surface, Z. Angew. Math. Phys., 2019, vol.70, no. 2, Art. 46, 9 pp.

[7] Kuzenov, V.V. and Ryzhkov, S. V., Calculation of Plasma Dynamic Parameters of the MagnetoInertial Fusion Target with Combined Exposure, Phys. Plasmas, 2019, vol. 26, no. 9, Art. 092704.

[8] Isakeev, A. I., Kiselyov, I. G., and Filatov, V. V., Efficient Methods of Cooling Power Semiconductor Devices, Leningrad: Energoizdat, 1982 (Russian).

[9] Dilevskaya, E. V., Kaskov, S. I., and Leontyev, A. I., The Vortex Investigation of Heat ExchangeUnconventional Method of Energy Efficiency Increasing of Chillers for Power Electronic Devices, Vestn. Mezhdunarodn. Akad. Kholoda, 2007, no.4(25), pp. 30-32 (Russian).

[10] Gortyshov, Yu. F., Isaev, S. A., Shchelchkov, A. V., Popov, I. A., and Mironov, A. A., Complex Studies of Vortex Heat Transfer Enhancement, in Proc. of the 6st Internat. Conf. "Heat and Mass Transfer and Hydrodynamics in Swirling Flows" (Novosibirsk, 2017), p. 48.

[11] Isaev, S. A. and Leontiev, A. I., Heat Transfer Enhancement on Structured Surfaces with Ordered Vortex Generators, in Proc. of the 6st Internat. Conf. "Heat and Mass Transfer and Hydrodynamics in Swirling Flows" (Novosibirsk, 2017), p. 18.

[12] Vortical Technologies for Power Engineering, A. I. Leontiev (Ed.), Moscow: MEI, 2017 (Russian).

[13] Rashidi, S., Hormozi, F., Sunden, B., and Mahia, O., Energy Saving in Thermal Energy Systems Using Dimpled Surface Technology: A Review on Mechanisms and Applications, Appl. Energy, 2019, vol. 250, pp. 1491-1547.

[14] Leontiev, A.I., Kaskov, S. I., Gortyshov, Yu.F., Shchelchkov, A. V., Popov, I. A., and Olimpiev, V. V., Hydrodynamics and Heat Transfer in Heat Exchanger Channels with Spherical Holes, in Proc. of the ASME International Mechanical Engineering Congress and Exposition "IMECE'2006 - Heat Transfer» (Chicago, 2006), Order No. I757DV.

[15] Leontiev, A.I., Kaskov, S.I., Gortyshov, Yu.F., Shchelchkov, A. V., Popov, I. A., and Olimpiev, V.V., Efficiency of Surface Heat Transfer Intensifiers for Laminar and Turbulent Flows in Heat Exchanger Channels, in Proc. of the ASME International Mechanical Engineering Congress and Exposition "Heat Transfer" (IMECE'2006, Chicago, 2006), Order No. I757DV.

[16] Khalatov, A.A., Heat Transfer and Hydrodynamics near Surface Dimples Holes, Kiev: Institute of Mathematics NAS of Ukraine, 2005 (Russian).

[17] Kiknadze, G.I., Gachechiladze, I.P., and Alekseev, V.V., Self-Organization of Tornado-Like Jets in Flows of Viscous Continua and Enhancement of Heat Transfer Which Accompanies This Phenomenon, Moscow: MEI, 2005 (Russian). 
[18] Bystrov, Yu. A., Isaev, S. A., Kudryavtsev, N. A., and Leontiev, A. I., Numerical Simulation of Vortex Heat Transfer Intensification in Tube Packages, Saint-Petersburg: Sudostroenie, 2005 (Russian).

[19] Zhou, W., Rao, Y., and Hu, H., An Experimental Investigation on the Characteristics of Turbulent Boundary Layer Flows over a Dimpled Surface, J. Fluids Eng., 2016, vol. 138, no. 2, 021204, 13 pp.

[20] Kopp, M. I., Tur, A. V., and Yanovsky, V. V., Nonlinear Dynamo Theory, Nelin. Dinam., 2015, vol. 11, no. 2, pp. 241-266 (Russian).

[21] Leontiev, A.I., Gortyshov, Yu. F., Olimpiev, V. V., Dilevskaya, E. V., Popov, I. A., Kaskov, S. I., and Shchelchkov, A. V., Development of Fundamental Foundations for Prototyping Energy-Efficient Heat Exchangers with Surface Intensification of Heat Exchange, in Proc. of the 4th Russian National Conf. on Heat Transfer (Moscow, 2006): Vol. 1, pp. 253-257 (Russian).

[22] Gortyshov, Iu. F., Popov, I. A., Olimpiev, V. V., Shchelchkov, A. V., and Kaskov, S. I., Thermohydraulic Efficiency of Perspective Methods of Heat Transfer Intensification in Heat Exchange Equipment Channels, Kazan: Center of Innovative Technologies, 2009 (Russian).

[23] Popov, I. A., Fluid Mechanics and Heat Transfer in Channels with Swirling and Vortex Flows and the Creation of High Performance Heat Exchangers, in Proc. of the 6st Internat. Conf. "Heat and Mass Transfer and Hydrodynamics in Swirling Flows" (Novosibirsk, 2017), p. 26.

[24] Zubkov, N. N., Nikitenko, S.M., Kaskov, S.I., and Nikitenko, M.S., Analysis of Arrangements of Heaters with Tubes Finned by Deforming Cutting, J. Eng. Thermophys., 2020, vol. 29, no.4, pp. 600-611.

[25] Shchukin, A. V., Kozlov, A.P., Agachev, R. S., and Chudnovsky, Ya. P., Intensification of Heat Exchange by Spherical Dimples under the Influence of Perturbing Factors, V. E. Alemasov (Ed.), Kazan: Kazan. Gos. Tekhn. Univ., 2003 (Russian).

[26] Turnow, J., Kornev, N., Isaev, S., and Hassel, E., Vortex Mechanism of Head Transfer Enhancement in a Channel with Spherical and Oval Dimples, Heat Mass Transf., 2011, vol. 47, no. 3, pp. 301-313.

[27] Chulyunin, A. Yu., Study of a Near Wall Flow Structures on a Surface of Deep Dimples, in Proc. of the 6st Internat. Conf. "Heat and Mass Transfer and Hydrodynamics in Swirling Flows" (Novosibirsk, 2017), p. 97.

[28] Popov, I. A., Shchelchkov, A.V., and Yarkaev, M.Z., Heat Transfer and Hydraulic Resistance in Channels with Spherical Protrusions, High Temp., 2016, vol. 54, no. 6, pp. 842-850.

[29] Zubkov, N. N. and Bityutskaya, Yu. L., Simulation of the Deformational Cutting and the Geometric Parameters of Pin Structures to Analyze the Thermohydraulic Characteristics of Heat-Removal Plates, Russ. Metall., 2018, vol. 2018, no. 13, pp. 1202-1207.

[30] Vinogradov, Yu. A., Zditovets, A. G., Egorov, K. S., Panova, O. I., and Strongin, M. M., Development of Virtual Stand for Research of Flows and Heat Transfer in the Plane-Parallel Channel with Dimpled Boundary Wall with Application on Modern CFD-Complex STAR-CD, in Proc. of the 16th School-Seminar of Young Scientists and Specialists under Supervision of Acad. A. I. Leontiev (SaintPetersburg, 2007): Vol. 2, pp. 394-396 (Russian).

[31] Egorov, K.S., Kaskov, S.I., and Panova, O. I., Effect of the Reynolds Number on the ThermalHydraulic Efficiency of the Hole Relief, in Proc. of the 3rd Russian National Conference "Future of Mechanical Engineering", Moscow: MGTU, 2010, pp. 170-171 (Russian).

[32] Al-Kharbavi, N. T. A., Popov, I. A., Shchelchkov, A. V., and Kaskov, S. I., Electronic Cooling Systems on Base of Enhanced Finned Heat Pipes, in Proc. of the 6th Russian National Conference on Heat Transfer (Moscow, 2014): Vol. 3, pp. 11-12.

[33] Popov, I. A., Al-Kharbavi, N. T. A., and Shchelchkov, A. V., Cooling Systems of Power Electronics on the Basis of the Edging Thermal Pipes with the Intensification of the Heat Emission, Energetika Tatarstana, 2014, no. 2(34), pp. 26-29 (Russian). 